\title{
Prenatal exposure to non-steroidal anti-inflammatory drugs (NSAIDs) and neurodevelopmental outcomes in children
}

Running title: Prenatal NSAIDs exposure and childhood neurodevelopment

Maja Markovic, ,a, Sonja A. Swanson, ${ }^{\text {b }}$ Bruno H. Stricker, ${ }^{\text {b,f, }}$ Vincent W. V. Jaddoe, ${ }^{\text {a,b,e }}$ Frank C. Verhulst, ${ }^{\mathrm{c}}$ Henning Tiemeier, ${ }^{\mathrm{b}, \mathrm{c}, \mathrm{d}}$ Hanan El Marroun ${ }^{\mathrm{a}, \mathrm{c}, \mathrm{e}, \mathrm{g}}$

a The Generation R Study Group, Erasmus University Medical Center, Rotterdam, The Netherlands

${ }^{\mathrm{b}}$ Department of Epidemiology, Erasmus Medical Center, Rotterdam, The Netherlands

c Department of Child and Adolescent Psychiatry, Erasmus Medical Center, Rotterdam, The Netherlands

${ }^{\mathrm{d}}$ Department of Psychiatry, Erasmus Medical Center, Rotterdam, The Netherlands

e Department of Pediatrics, Erasmus Medical Center, Rotterdam, The Netherlands

${ }^{\mathrm{f}}$ Inspectorate of Healthcare, The Hague, The Netherlands

${ }^{\text {g }}$ Department of Psychology, Education and Child Studies - Erasmus University Rotterdam

\section{Correspondence:}

Hanan El Marroun, E-mail: hmarrounel@erasmusmc.nl, Address: Department of Child and Adolescent Psychiatry, Erasmus Medical Centre, Wytemaweg 80 - KP2, 3015 CN Rotterdam, The Netherlands, Tel: +31 (0) 10-7034275

Keywords: Anti-Inflammatory Agents Non-Steroidal, confounding factors, behavior, bias, cognition, prenatal exposure delayed effects 


\section{Key points:}

- Little is known about the effect of prenatal exposure to non-steroidal anti-inflammatory drugs (NSAIDs) on child development.

- It is important to address sources of potential confounding in pharmacoepidemiologic studies that investigate prenatal drug exposure and subsequent child development.

- This study investigated sources of confounding by controlling for several potential confounders, using outcomes measured repeatedly and across different informants, studying pre-pregnancy exposure, and using proposed negative outcomes.

- The association of prenatal exposure to NSAIDs and child attention problems is possibly partly explained by residual confounding by indication and measurement-related biases.

- Future studies may consider using more objective measurements of outcomes alongside richer assessments of women's decisions to use NSAIDs prior to and during pregnancy.

Word count main text: 3348

Word count Abstract: 250 words

Prior postings and presentations: This work has not been presented or printed elsewhere. 


\section{Abstract}

Purpose: Non-steroidal anti-inflammatory drugs (NSAIDs) are commonly used during pregnancy. Findings that prenatal NSAID exposure may affect offspring neurodevelopment have been inconsistent. We investigated the effect of prenatal NSAID exposure on childhood neurodevelopment, and explored the susceptibility of our effect estimates to forms of bias via negative exposure, negative outcome, and multi-informant analyses.

Methods: In a cohort of pregnant women ( $\mathrm{n}=6876)$, perinatal NSAID use was assessed by prescriptions and self-report. Primary neurodevelopmental outcomes included attention problems using maternal reports at $1 \frac{1 / 2,}{2} 3$ and 5 years. To explore potential systematic biases, we compared estimates from maternally reported attention problems to a teacher's report and a measure of nonverbal intelligence assessed at a clinic visit at age 6 years; we also used NSAID use before pregnancy and somatic problems as a "negative” exposure and outcome, respectively.

Results: Maternal reports suggested that prenatal exposure to NSAIDs was associated with more attention problems at younger ages (e.g., at age 3: mean difference in attention problems score: 0.30; $95 \%$ CI $0.12,0.48)$. However, no strong association with attention problems was found in the teacher report, and a similarly strong association between prenatal NSAID exposure and somatic complaints suggests residual confounding by indication likely remains. Moreover, prenatal exposure to NSAIDs was not associated with an observed measure of IQ (mean difference in IQ score: -0.32; 95\% CI: -1.82, 1.19).

Conclusions: Jointly, our results suggest that the observed associations between prenatal exposure to NSAIDs and child attention problems reflect systematic biases of a null or small effect. 


\section{Introduction}

Medication use during pregnancy is common. ${ }^{1}$ One of the most frequently prescribed medications during pregnancy is non-steroidal anti-inflammatory drugs (NSAIDs). ${ }^{2-6}$ Over-thecounter use of NSAIDs is widespread, especially during the first trimester of pregnancy. ${ }^{7,8}$ As medication use during pregnancy may pose a risk to the mother and her developing fetus, the potential benefits of the medication must be weighed against the risks for both mother and child. Therefore, information to guide patients and physicians to make a well-informed decision for the appropriate treatment during pregnancy is needed.

NSAIDs, for example ibuprofen, naproxen, diclofenac and aspirin (acetylsalicylic acid), ${ }^{9}$ are widely recognized for their analgesic, antipyretic, and anti-inflammatory effects. ${ }^{10}$ The action of NSAIDs results from cyclooxygenase (COX) inhibition, followed by suppression of prostaglandins-synthesis in the brain. ${ }^{11}$ Based on animal studies, COX-2 has been shown to be present in the brain and seems to play an essential role in neurodevelopment. ${ }^{12,13}$ Thus, a key question is whether prenatal NSAID use has effects on foetal brain development.

Earlier research examining the association between prenatal exposure to NSAIDs and cognitive impairment in children has been inconsistent, with studies finding negative ${ }^{14}$ and positive ${ }^{15}$ associations with intelligence measures and cognitive functioning. For behavioural outcomes such as Attention Deficit Hyperactivity Disorder (ADHD) and attention problems, results have likewise been inconsistent. One animal study reported a protective effect on the risk of behavioural difficulties and hyperactivity. ${ }^{16}$ In epidemiologic studies, some studies have found no association ${ }^{17}$ while others have reported higher risks of attention problems among children exposed to NSAIDs in utero. ${ }^{14}$ 
Discrepancies in previous research may be explained by: small sample sizes; differences in study populations (e.g. one study restricted to children born preterm ${ }^{17}$ ); and differing susceptibilities to biases such as residual confounding by indication, ${ }^{18}$ and artefacts of the study design (e.g., retrospective versus prospective ${ }^{14}$ studies), or available measures for medication use (e.g., self-report versus prescription) or outcome (e.g., parent report versus other informants). In the absence of randomized trials, a crucial challenge faced by all these studies is disentangling the neurodevelopmental consequences of prenatal NSAIDs exposure from the effects of the underlying indication for drug use (i.e., confounding by indication). ${ }^{19}$ Unlike more specialized prescription drugs with well-defined indications for use, analgesics are used for a wide range of indications. ${ }^{18}$ Hence, confounders can be especially difficult to define, measure and appropriately adjust for.

The aim of the current study is to investigate the effect of prenatal exposure to NSAIDs and neurodevelopment of children. We begin by estimating effects on neurodevelopmental outcomes while adjusting for many sources of potential confounding. To explore possible systematic biases to our estimates and prior studies' estimates, we compared our estimates from maternally-reported outcomes to a teacher's report, and further used NSAID use before pregnancy and somatic problems as a "negative" exposure and outcome, respectively. ${ }^{20}$ 


\section{Methods}

\section{Study design and Participants}

This study was embedded in the Generation R Study, a population-based prospective cohort from

foetal life onwards. ${ }^{21,22}$ Briefly, all pregnant women living in Rotterdam, the Netherlands, with an expected delivery date between April 2002 and January 2006 were invited to participate. The overall response rate based on the number of children born in Rotterdam in the same period was $61 \% .{ }^{23}$ The study was conducted in accordance with the guidelines of the World Medical Association Declaration of Helsinki and was approved by the Medical Ethics Committee of the Erasmus Medical Center, Rotterdam. Written informed consent was obtained from all participants.

In total, 8237 children in the pre- and postnatal follow-up period were eligible. Those with missing or incomplete data on prenatal NSAID exposure were excluded $(\mathrm{N}=716)$. Of the remaining 7521 mother-child dyads with baseline data, 645 participants did not have information on cognitive, emotional or behavioural functioning (of at least one assessment) and were therefore excluded, yielding a final study sample size of 6876 (91.4\%) children and mothers. The number of children varies slightly per analyses (Supplementary Figure S1).

\section{Measurements}

Prenatal NSAID exposure

NSAID use during pregnancy was assessed via both self-report and prescription records. In each trimester, pregnant mothers reported in questionnaires whether they had used any medication. In the first trimester, the mothers were asked whether they used medications within the past 6 
months. The mothers filled out the type of medication and when it was used (during pregnancy, only before pregnancy, or stopped when I knew I was pregnant). In the second and third trimesters, we asked which medications were used in the preceding 3 months. From these questionnaires, we assessed NSAIDs exposure and timing (before or during pregnancy). One question with several open text fields was used and thus information was not systematically collected. Further, no additional methods were used to enhance medication recall during pregnancy.

We asked women for permission to contact their pharmacy to obtain information on filled prescriptions. For the large majority, permission was obtained and data were requested, but prescription records were only available in $60.2 \%(n=4142)$ of our study sample due to a delay in linkage (this specific data collection started later and could not be retrieved in all participants). The records screened for NSAIDs use provided information on the type of NSAIDs, duration, and dose. The agreement between self-reports and prescription records was $64.8 \%$; the majority of disagreements were women who self-reported use while having no filled prescription, which we interpreted as likely indicating over-the-counter use. Based on either the prescription or selfreported measures indicating use, there were 5117 (74.4\%) women who did not use NSAIDs before or during pregnancy, 1286 (18.7\%) with NSAID use before but not during pregnancy, and 473 (6.9\%) with NSAID use during pregnancy.

\section{Behavioural and emotional problems}

At the age of 11/2, 3 and 5 year, child problem behaviour was assessed using the Dutch version of the Child Behavior Checklist for ages 11/2 - 5 years (CBCL/11/2 -5). ${ }^{24}$ The CBCL/11/2 -5 is a 99item parent report on problem behaviours with well-established psychometric properties. ${ }^{25}$ The 
Dutch version of the CBCL/11/2 -5 is reliable and well validated, ${ }^{26}$ and the subscales had a good fit in 23 international studies across diverse societies. ${ }^{27}$

The current study focused on the CBCL subscales for attention problems and somatic complaints. The CBCL has good reliability and validity; the internal consistency of the attention

problems and somatic complaints scale, measured by Cronbach's alpha was 0.68 and $0.80 .^{24}$ Attention problems were also rated by teachers using the Teacher's Report Form (TRF) ${ }^{25}$ when the children were aged 6 years. Although $40 \%$ of children were 6 years or older at the time of assessment, we used the CBCL 1.5-5 (preschool version) for reasons of continuity.

Nonverbal intelligence

We used the Snijders-Oomen Non-verbal Intelligence Test 2.5-7-Revised (SON-R 2.5-7), ${ }^{28}$ a reliable and valid measure. ${ }^{28-30}$ Two subtests of the SON-R 2.5-7 were administered in Generation R at age 6 years: Mosaics, assessing spatial visualization abilities, and Categories, assessing abstract reasoning abilities. A scaled total score can be calculated for any combination of subtests with the same distribution characteristics as the IQ score. A correlation of $r=0.86$ was found between the score derived from the Mosaics and Categories subsets, and the IQ scores derived from the complete test. ${ }^{28}$ The raw scores of each subtest were standardized; the sum of the standardized scores were converted into SON-R IQ score using age-specific reference scores provided in the manual (mean=100, $S D=15$ ). The average reliability of the SON-R $2 \frac{1}{2} 7$ IQ score is 0.90 , range $0.86-0.92$ for the respective age. ${ }^{28}$ The reliability of the subtests that were used in our study is: 0.73 for mosaics and 0.71 for categories. We chose a validated Dutch instrument and specifically investigated non-verbal IQ, because our sample is multi-ethnic 
(differences in exposure to the Dutch language in young children could be present), and bilingualism is common.

\section{Covariates}

Potential confounders were selected based on the previous literature. ${ }^{14-17,31,32}$ Information on gender, birth weight, and Apgar score at 5 min after birth were obtained from hospital records. Gestational age was obtained by foetal ultrasound examination. Maternal body mass index was determined by weight and height at the first visit. Maternal age, ethnicity and education were assessed by questionnaires. Ethnicity was defined as Dutch, non-Dutch Western and non-Dutch non-Western. Maternal education defined by the highest completed education was classified as "higher" (higher vocational training or higher academic education), "secondary" (more than 3 years general secondary school), and "primary" (lower vocational training or 3 years general secondary school). Maternal tobacco smoking was obtained in each trimester. On the basis of all three questionnaires, we defined the following categories: "never smoked," "smoked until pregnancy was known," and "continued throughout pregnancy". Maternal alcohol use was categorized into "never drank in pregnancy”, “drank until pregnancy was known”, “continued to drink occasionally" and "continued to drink frequently". Monthly household income at enrolment was categorized into $>€ 2000$ (more than modal income), €1200-€2000, and $<€ 1200$ (below social security level). Maternal cognitive ability was assessed during the visit to the research centre at child's age 5-7 years, with a computerized version of the Ravens Advanced Progressive Matrices (APM) Test, set I. ${ }^{33}$ Set I consists of 12 items and has been shown to be a reliable and valid short form of the Raven’s Progressive Matrices to asses nonverbal intelligence. 
${ }^{34}$ Maternal psychiatric symptoms were assessed at 20 weeks of pregnancy and when the child was 3 years old using the Brief Symptom Inventory (BSI), a validated self-report questionnaire. 35,36 At 20 weeks of pregnancy the complete 53-item questionnaire was assessed, which contained the following subscales: depression, anxiety, phobic anxiety, hostility, interpersonal sensitivity, paranoid ideation, somatization, obsessive-compulsive behaviour and psychoticism. We computed the Global Severity Index (GSI), ${ }^{35}$ which we defined as the average item score across the two time points. Like NSAIDs, use of antidepressants (selective serotonin reuptake inhibitors) and benzodiazepines was also collected using a combination of questionnaires and pharmacy records. Further, as use of NSAIDs may co-occur with other medications, such as paracetamol (a.k.a., acetaminophen), we also collected this information from the same questionnaires (but not pharmacy records).

\section{Statistical analysis}

We estimated the associations between prenatal NSAIDs use and the following outcomes using separate linear regression models: maternal reports of somatic complaints and attention problems at 1.5, 3, and 5 years; teaching reports of attention problems at 6 years and non-verbal intelligence at 5 years. Models were adjusted for age and gender of the child; ethnicity, age, education and psychopathology of the mother; smoking and alcohol consumption during pregnancy; APM score of the mother; and exposure to paracetamol, antidepressants and benzodiazepines. For comparison, we also present unadjusted associations.

We used NSAIDs use before pregnancy as a negative exposure and we used somatic complaints as a negative outcome in order to detect (residual) confounding. ${ }^{20}$ It could be 
possible that maternal reports of child behaviour may be biased via the personal characteristics of the respondent ${ }^{37}$ and to account for this potential bias, we used teacher reports of attention problems and used a test-battery as a more objective measured outcome.

The missing values on all confounders were below $20 \%$ except for the maternal cognitive ability score (22.4\%). We accounted for missing information on the confounders by using the Markov Chain Monte Carlo imputation with predictive mean matching for continuous variables. ${ }^{38}$ Ten imputed datasets were generated using a fully conditional specified model. Analyses were performed separately on each completed dataset and thereafter combined to one pooled estimate. Children who did not have complete data on cognitive, emotional, or behavioural outcomes were more likely to have a lower birth weight and have mothers who were lower educated, younger, more likely to be non-Dutch, smoked more frequently, and had higher psychopathology symptom scores (Supplemental Table 1). 


\section{Results}

\section{Characteristics of the study population}

The maternal and child characteristics stratified by prenatal NSAID exposure (most commonly ibuprofen, naproxen, diclofenac and aspirin) are presented in Table 1. Compared to mothers who did not use NSAIDs during pregnancy, mothers who used NSAIDs during pregnancy had a slightly higher body mass index and were more likely to smoke, use alcohol, and have psychopathology symptoms during pregnancy. Mothers who used NSAIDs before pregnancy were more likely to be non-Dutch, more likely to have a lower income and were less educated and had a lower cognitive ability score, as compared to the mothers who did not use NSAIDs.

Insert Table 1 here

NSAIDs exposure during pregnancy and maternal report of child attention problems

Relative to mothers who did not use NSAIDs, mothers who used NSAIDs during pregnancy reported more attention problems in their children at ages 1.5, 3, and 5 years (Table 2). Adjusting for covariates slightly attenuated these estimates, but robust associations remained present at ages 1.5 years (mean difference in score: 0.27; 95\% CI 0.07, 0.46) and 3 years (mean difference in score: 0.30; 95\% CI 0.12, 0.48).

Insert Table 2 here

Investigation of residual confounding: pre-pregnancy NSAID use as a "negative exposure"

Pre-pregnancy NSAID use was not strongly associated with maternally reported attention problems at ages 1.5, 3, and 5 years (Table 3). 
Insert Table 3 here

Investigation of residual confounding: somatic complaints as a "negative outcome"

Prenatal NSAIDs exposure was not strongly associated with maternal report of somatic complaints (Table 4). For example, the adjusted mean difference in the somatic complaints score at age 3 years was $0.19(95 \% \mathrm{CI}-0.01,0.38)$.

Insert Table 4 here

Investigation of reporting bias: teacher report of attention problems

In contrast to maternal report of attention problems, no strong association was seen between prenatal NSAID exposure and teacher-reported attention problems at age 6 years (mean difference in attention score: -0.24 ; $95 \%$ CI $-1.23,0.76$ ). In addition, no strong association was observed between prenatal NSAID exposure and teacher-reported somatic problems at 6 years (mean difference in somatic score: -0.05; 95CI -0.17, 0.08).

Investigation of reporting bias: observed measure of non-verbal intelligence

As shown in Table 5, relative to no exposure, prenatal NSAIDs exposure had little observed association with nonverbal intelligence (mean difference in IQ score: -0.07; 95\% CI -1.67, 1.53). Adjusting for several potential confounders did not materially change the estimate (-0.32; $95 \%$ CI -1.82, 1.19).

Insert Table 5 here 


\section{Discussion}

In the present study, mothers reported that children prenatally exposed to NSAIDs had a higher risk of attention problems (particularly at younger ages). Alongside our results regarding maternal reports of somatic complaints, maternal reports of pre-pregnancy NSAIDs use, teacher reports of attention problems and somatic complaints together and an observational measure of non-verbal intelligence, these findings altogether suggest that this apparent association can perhaps be largely explained by residual confounding by indication and measurement-related biases. Below we detail the insights gained from each of these additional analyses.

Confounding by indication is of particular concern in studying NSAID use during pregnancy, as the reasons for using these medications may affect child neurodevelopment and reporting of child neurodevelopment directly. As NSAIDs are often used to treat fever, pain and inflammation, it could be that these underlying conditions could be risk factors for neurodevelopmental problems. ${ }^{39}$ While we were able to adjust for a rich set of measures on maternal psychopathology and other characteristics, apparent associations may still be explained by residual confounding. We used "negative" exposures and outcomes to indirectly assess whether confounding remained. As prenatal NSAID use and somatic complaints are likely confounded by the same sources of confounding as our primary research question, yet biologically we expect no direct effect, the fact that we found similar effect estimates (in the same direction) even after adjusting for measured confounders may suggests that the effect estimates may be biased. On the other hand, studying pre-pregnancy NSAID use and attention problems, which likewise we would biologically expect no direct effect, we did not find a robust association and the effect estimates were in the opposite direction. One possible reason for this is that the confounding structure may actually be different, as the set and severity of indications and 
the choice for using NSAIDs pre-pregnancy may vary substantially from what occurs during pregnancy. In other words, pre-pregnancy NSAID use may not serve as a suitable "negative" exposure after all; there is some evidence of this in the descriptive statistics in Table 1 , as mothers who reported pre-pregnancy NSAID use differed from mothers who reported used during pregnancy in terms of education, family income and other health behaviours during pregnancy.

Beyond confounding by indication, another possible limitation of studying maternally reported attention problems as an outcome is information bias. Namely, indications for treatment and other maternal characteristics may affect the measurement of the outcome while not directly affecting attention problems themselves. To assess this possibility, we considered outcomes reported by other sources, namely teacher-reported attention problems and a non-verbal cognitive functioning test performed at our research centre. We found no association between prenatal NSAIDs exposure and attention problems when the teachers reported about the child. The teachers' perspective is valuable since teachers observe child behaviour in task-oriented situations where children are required to concentrate. Moreover, teachers can easily compare a particular child's behaviour with that of a relatively large group of classmates of the same developmental level. ${ }^{40}$ Therefore, our findings may suggest that mothers might over-report children's attention problems, and that it is important to use a multi-informant approach as information of different informants may reflect variations in children's behavior across diverse settings and relational circumstances. ${ }^{41-43}$ This finding is likewise corroborated by the null findings for the non-verbal intelligence score, although this score is measuring a different cognitive construct and thus can only be seen as indirect evidence of information bias. 
However, there is a possibility that these associations of prenatal NSAIDs exposure on attention are transient being prominent in early childhood, as maternal NSAIDs use was not related to maternal reported attention problems as 5 years and our only available external reports (teacher reports; IQ score) were measured at 6 years.

Strengths of the current study included its prospective design and use of multiple informants and multiple, repeated assessments. Some limitations of our study in particular merit consideration. First, while our analyses support that biases may explain much of the apparent association, our evidence only indirectly addresses the magnitude of bias. As such, it is possible that small (but perhaps clinically inconsequential) effects would remain even if such biases could be fully corrected for. Future studies may consider extending the set of analyses we have conducted here with more objective measurements of outcomes alongside richer assessments of women's decisions to use NSAIDs prior to and during pregnancy. Second, as is common in birth cohort studies, some selective loss to follow-up among families from low socioeconomic status and non-Western origin occurred in Generation R. ${ }^{21}$ While our study focused on exploring confounding and information biases, future work may consider the role of selection bias due to loss to follow-up. Further, in this study we did not examine the effects of different type of NSAIDs, dose dependent effects and trimester-specific effects, as the information that we collected from the questionnaires was very general. However, NSAIDS all involve COXinhibition, followed by suppression of prostaglandin synthesis in the brain, ${ }^{11}$ and thus typespecific effects would be unlikely. Further, somatic complaints were asked using the CBCL, this subscale specifically refers to physical problems (e.g. headaches, nausea and stomach aches) without a known medical cause; and thus we cannot exclude that part of these somatic complaints are due to underlying psychopathology. Finally, the analyses were performed using a 
variety of measures at different ages in slightly different samples; differences in the study populations included in each analysis or differences in their susceptibility to bias due to loss to follow up may theoretically explain some results. and thus, we need to be cautious in the interpretations of our findings.

In conclusion, by controlling for several potential confounders, using outcomes measured repeatedly and across different informants, studying pre-pregnancy exposure, and using proposed negative outcomes, this study provides evidence that the observed associations between prenatal exposure to NSAIDs and child attention problems in this study and possibly previous studies likely indicate null or small (i.e. near-null) causal effect.

\section{Acknowledgements \& Funding}

The Generation R Study is conducted by the Erasmus Medical Centre in close collaboration with the Faculty of Social Sciences and School of Law of the Erasmus University Rotterdam, the Municipal Health Service, Rotterdam, the Rotterdam Homecare Foundation, and the Stichting Trombosedienst \& Artsenlaboratorium Rijnmond, Rotterdam. We gratefully acknowledge the contribution of general practitioners, hospitals, midwives, and pharmacies in Rotterdam.

The European Commission financed an ERAWEB scholarship grant, which was granted to Maja Markovic (grant agreement 2013-2548/001-001-EMA2). In addition, the Erasmus University Rotterdam granted Dr. El Marroun a personal fellowship (EUR Fellow 2014) and supported this work financially. The Netherlands Organization for Scientific Research (NWO Brain \& Cognition Program Grant 433-09-311 and VIDI Grant 017.106.370) also supported this work. Dr. Swanson was supported by a NWO/ZonMW Veni grant [91617066]. The first phase of the 
Generation R Study is made possible by financial support from the Erasmus Medical Centre, and the Netherlands Organization for Health Research and Development (ZonMW Geestkracht Program 10.000.1003) the European Union's Horizon 2020 research and innovation program under grant agreement No.633595 (DynaHEALTH) and No.733206 (LifeCycle). Funding agencies had no role in the design and conduct of the study, collection, management, analyses and interpretation of the data; and preparation, review or approval of the manuscript and the decision to submit it for publication. 


\section{References}

1. Lee E, Maneno MK, Smith L, et al. National patterns of medication use during pregnancy. Pharmacoepidemiol Drug Saf. 2006;15(8):537-545.

2. Bloor M, Paech M. Nonsteroidal anti-inflammatory drugs during pregnancy and the initiation of lactation. Anesth Analg. 2013;116(5):1063-1075.

3. Ostensen ME, Skomsvoll JF. Anti-inflammatory pharmacotherapy during pregnancy. Expert Opin Pharmacother. 2004;5(3):571-580.

4. Bakker MK, Jentink J, Vroom F, Van Den Berg PB, De Walle HE, De Jong-Van Den Berg LT. Drug prescription patterns before, during and after pregnancy for chronic, occasional and pregnancy-related drugs in the Netherlands. BJOG. 2006;113(5):559-568.

5. Ericson A, Kallen BA. Nonsteroidal anti-inflammatory drugs in early pregnancy. Reprod Toxicol. 2001;15(4):371-375.

6. Olesen C, Steffensen FH, Nielsen GL, de Jong-van den Berg L, Olsen J, Sorensen HT. Drug use in first pregnancy and lactation: a population-based survey among Danish women. The EUROMAP group. Eur J Clin Pharmacol. 1999;55(2):139-144.

7. Glover DD, Amonkar M, Rybeck BF, Tracy TS. Prescription, over-the-counter, and herbal medicine use in a rural, obstetric population. Am J Obstet Gynecol. 2003;188(4):1039-1045.

8. Cleves MA, Savell VH, Jr., Raj S, et al. Maternal use of acetaminophen and nonsteroidal antiinflammatory drugs (NSAIDs), and muscular ventricular septal defects. Birth Defects Res A Clin Mol Teratol. 2004;70(3):107-113.

9. Green GA. Understanding NSAIDs: from aspirin to COX-2. Clin Cornerstone. 2001;3(5):50-60.

10. Litalien C, Jacqz-Aigrain E. Risks and benefits of nonsteroidal anti-inflammatory drugs in children: a comparison with paracetamol. Paediatr Drugs. 2001;3(11):817-858.

11. Hilario MO, Terreri MT, Len CA. Nonsteroidal anti-inflammatory drugs: cyclooxygenase 2 inhibitors. J Pediatr (Rio J). 2006;82(5 Suppl):S206-212.

12. Kaufmann WE, Worley PF, Taylor CV, Bremer M, Isakson PC. Cyclooxygenase-2 expression during rat neocortical development and in Rett syndrome. Brain Dev. 1997;19(1):25-34.

13. Yamagata K, Andreasson KI, Kaufmann WE, Barnes CA, Worley PF. Expression of a mitogeninducible cyclooxygenase in brain neurons: regulation by synaptic activity and glucocorticoids. Neuron. 1993;11(2):371-386.

14. Streissguth AP, Treder RP, Barr HM, et al. Aspirin and acetaminophen use by pregnant women and subsequent child IQ and attention decrements. Teratology. 1987;35(2):211-219.

15. Klebanoff MA, Berendes HW. Aspirin exposure during the first 20 weeks of gestation and IQ at four years of age. Teratology. 1988;37(3):249-255.

16. Bronson SL, Bale TL. Prenatal stress-induced increases in placental inflammation and offspring hyperactivity are male-specific and ameliorated by maternal antiinflammatory treatment. Endocrinology. 2014;155(7):2635-2646.

17. Marret S, Marchand L, Kaminski M, et al. Prenatal low-dose aspirin and neurobehavioral outcomes of children born very preterm. Pediatrics. 2010;125(1):e29-34.

18. Signorello LB, McLaughlin JK, Lipworth L, Friis S, Sørensen HT, Blot WJ. Confounding by indication in epidemiologic studies of commonly used analgesics. American journal of therapeutics. 2002;9(3):199-205.

19. Brandlistuen RE, Ystrom E, Eberhard-Gran M, Nulman I, Koren G, Nordeng H. Behavioural effects of fetal antidepressant exposure in a Norwegian cohort of discordant siblings. Int $J$ Epidemiol. 2015;44(4):1397-1407.

20. Lipsitch M, Tchetgen Tchetgen E, Cohen T. Negative controls: a tool for detecting confounding and bias in observational studies. Epidemiology. 2010;21(3):383-388. 
21. Jaddoe VW, van Duijn CM, Franco OH, et al. The Generation R Study: design and cohort update 2012. European journal of epidemiology. 2012;27(9):739-756.

22. Tiemeier H, Velders FP, Szekely E, et al. The Generation R Study: a review of design, findings to date, and a study of the 5-HTTLPR by environmental interaction from fetal life onward. $J$ Am Acad Child Adolesc Psychiatry. 2012;51(11):1119-1135 e1117.

23. Kooijman MN, Kruithof CJ, van Duijn CM, et al. The Generation R Study: design and cohort update 2017. Eur J Epidemiol. 2016;31(12):1243-1264.

24. Achenbach TM, Rescorla LA. ASEBA preschool forms \& profiles. University of Vermont, Research Center for Children, Youth and Families, Burlington, VT. 2000.

25. Achenbach TM, Rescorla L. ASEBA school-age forms \& profiles. Aseba Burlington; 2001.

26. Tick NT, van der Ende J, Koot HM, Verhulst FC. 14-year changes in emotional and behavioral problems of very young Dutch children. J Am Acad Child Adolesc Psychiatry. 2007;46(10):13331340.

27. Ivanova MY, Achenbach TM, Rescorla LA, et al. Preschool psychopathology reported by parents in 23 societies: testing the seven-syndrome model of the child behavior checklist for ages 1.5-5. $J$ Am Acad Child Adolesc Psychiatry. 2010;49(12):1215-1224.

28. Tellegen PJ, Winkel M, Wijnberg-Williams BJ, Laros JA. Snijders-Oomen Niet-Verbale Intelligentietest SON-R 2, 5-7. Handleiding [Snijders-Oomen Nonverbal Intelligence Test SONR 2, 5-7. Manual]. Hogrefe, Gottingen. 2005.

29. Jenkinson J, Roberts S, Dennehy S, Tellegen P. Validation of the Snijders-Oomen Nonverbal Intelligence Test-Revised 21/2-7 for Australian Children with Disabilities. Journal of Psychoeducational Assessment. 1996;14(3):276-286.

30. Moore C, O'Keefe SL, Lawhon D, Tellegen P. CONCURRENT VALIDITY OF THE SNIJDERS-OOMEN NONVERBAL INTELLIGENCE TEST 2V2-7-REVISED WITH THE WECHSLER PRESCHOOL AND PRIMARY SCALE OF INTELLIGENCE-REVISED. Psychological reports. 1998;82(2):619-625.

31. Amin SB, Kamaluddeen M, Sangem M. Neurodevelopmental outcome of premature infants after exposure to antenatal indomethacin. Am J Obstet Gynecol. 2008;199(1):41 e41-48.

32. Fritz H, Suter HP. Postnatal development of young rats following the treatment of the dams with sodium salicylate during late periods of pregnancy. Arzneimittelforschung. 1985;35(6):937-939.

33. Prieler J. Raven's advanced progressive matrices. Schufried, Mödling, Austria. 2003.

34. Chiesi F, Ciancaleoni M, Galli S, Primi C. Using the Advanced Progressive Matrices (Set I) to assess fluid ability in a short time frame: An item response theory-based analysis. Psychological assessment. 2012;24(4):892.

35. de Beurs E. Brief symptom inventory. Handleiding Leiden (Netherlands): Pits Publishers. 2004.

36. Derogatis LR, Spencer P. Brief symptom inventory: BSI. Pearson Upper Saddle River, NJ, USA:; 1993.

37. Najman JM, Williams GM, Nikles J, et al. Bias influencing maternal reports of child behaviour and emotional state. Soc Psychiatry Psychiatr Epidemiol. 2001;36(4):186-194.

38. Sterne JAC, White IR, Carlin JB, et al. Multiple imputation for missing data in epidemiological and clinical research: potential and pitfalls. Bmj. 2009;338:b2393.

39. Cordeiro CN, Tsimis M, Burd I. Infections and Brain Development. Obstet Gynecol Surv. 2015;70(10):644-655.

40. Schweitzer JB, Cummins TK, Kant CA. Attention-deficit/hyperactivity disorder. Medical Clinics of North America. 2001;85(3):757-777.

41. Achenbach TM, McConaughy SH, Howell CT. Child/adolescent behavioral and emotional problems: implications of cross-informant correlations for situational specificity. Psychol Bull. 1987;101(2):213-232.

42. De Los Reyes A, Augenstein TM, Wang M, et al. The validity of the multi-informant approach to assessing child and adolescent mental health. (1939-1455 (Electronic)). 
43. Stanger C, Lewis M. Agreement among parents, teachers, and children on internalizing and externalizing behavior problems. Journal of Clinical Child Psychology. 1993;22(1):107-116. 
Table 1.Maternal and child characteristics stratified by prenatal NSAID exposure

\begin{tabular}{|c|c|c|c|}
\hline & \multicolumn{3}{|c|}{ Prenatal NSAID exposure } \\
\hline & $\begin{array}{l}\text { No use } \\
(\mathrm{n}=5117)\end{array}$ & $\begin{array}{l}\text { Use before pregnancy } \\
(\mathrm{n}=1286)\end{array}$ & $\begin{array}{l}\text { Use during pregnancy } \\
(\mathrm{n}=473)\end{array}$ \\
\hline \multicolumn{4}{|l|}{ Child characteristics } \\
\hline Gender (\% girls) & 50.15 & 49.92 & 50.32 \\
\hline Birth weight in grams, mean (SD) & 3409.43(575.78) & $3416.84(563.64)$ & $3377.08(535.00)$ \\
\hline $\begin{array}{l}\text { Gestational age at birth in weeks, mean } \\
\text { (SD) }\end{array}$ & $39.84(1.81)$ & $39.80(1.91)$ & $39.72(1.65)$ \\
\hline $\begin{array}{l}\text { Apgar score at } 5 \text { min after birth, mean } \\
\text { (SD) }\end{array}$ & $9.62(0.76)$ & $9.58(0.90)$ & $9.60(0.78)$ \\
\hline \multicolumn{4}{|l|}{ Maternal characteristics } \\
\hline Age in years, mean (SD) & $30.29(5.11)$ & $29.69(5.17)$ & 30.35 (4.83) \\
\hline \multicolumn{4}{|l|}{ Ethnicity (\%) } \\
\hline Dutch & 54.06 & 45.75 & 58.10 \\
\hline Non-Dutch Western & 9.00 & 6.90 & 8.71 \\
\hline Non-Dutch non-Western & 36.94 & 47.35 & 33.19 \\
\hline \multicolumn{4}{|l|}{ Education (\%) } \\
\hline Higher & 47.65 & 33.68 & 42.85 \\
\hline Secondary & 42.70 & 53.27 & 46.83 \\
\hline Primary & 9.65 & 13.05 & 10.32 \\
\hline \multicolumn{4}{|l|}{ Family income (\%) } \\
\hline$>2000 € /$ month & 62.28 & 51.95 & 60.42 \\
\hline $1200-2000$ €/month & 18.36 & 22.93 & 18.67 \\
\hline$<1200 € /$ month & 19.36 & 25.12 & 20.91 \\
\hline $\mathrm{BMI}$ in $\mathrm{kg} / \mathrm{m}^{2}$, mean $(\mathrm{SD})$ & $24.56(4.34)$ & $25.43(4.78)$ & $25.03(4.49)$ \\
\hline \multicolumn{4}{|l|}{ Smoking during pregnancy (\%) } \\
\hline Never & 75.62 & 70.25 & 66.13 \\
\hline Until pregnancy was known & 8.69 & 8.98 & 12.60 \\
\hline Continued throughout pregnancy & 15.69 & 20.77 & 21.27 \\
\hline \multicolumn{4}{|l|}{ Alcohol use during pregnancy (\%) } \\
\hline Never & 45.35 & 53.00 & 39.64 \\
\hline Until pregnancy was known & 13.22 & 12.16 & 16.03 \\
\hline Continued occasionally & 33.10 & 29.41 & 33.15 \\
\hline Continued frequently & 8.33 & 5.43 & 11.18 \\
\hline Psychopathology symptoms, mean (SD) & $0.28(0.36)$ & $0.34(0.41)$ & $0.35(0.41)$ \\
\hline Cognitive ability score, mean (SD) & $96.17(15.18)$ & $92.68(15.59)$ & $95.75(14.77)$ \\
\hline \multicolumn{4}{|l|}{ Co-medication (\%) } \\
\hline Antidepressants & 1.04 & 1.6 & 1.9 \\
\hline Benzodiazepines & 0.86 & 2.6 & 4.0 \\
\hline Paracetamol & 21.6 & 25.0 & 44.0 \\
\hline
\end{tabular}

SD: standard deviation, BMI: body mass index 


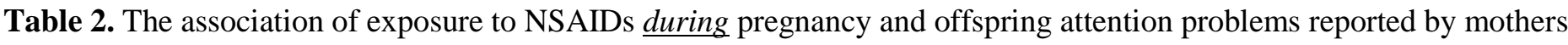

\begin{tabular}{llcr}
\hline Attention Problems & $\begin{array}{l}\text { N Exposed/ N Non- } \\
\text { exposed }\end{array}$ & $\begin{array}{l}\text { Unadjusted Mean Difference } \\
\text { in Attention Problems Score }\end{array}$ & $\begin{array}{c}\text { Adjusted Mean Differe } \\
\text { Attention Problems Scor }\end{array}$ \\
Maternal report at 1.5 yrs. & $336 / 4054$ & $0.31(0.11,0.51)$ & $0.27(0.07,0.46)$ \\
Maternal report at 3 yrs. & $310 / 3800$ & $0.35(0.16,0.53)$ & $0.30(0.12,0.48)$ \\
Maternal report at 5 yrs. & $372 / 4872$ & $0.13(-0.06,0.31)$ & $0.05(-0.13,0.23)$
\end{tabular}

† Adjusted model, covariates: age and gender of the child, ethnicity, age, education and psychopathology of the mother, smoking and alcohol consumption during pregnancy, and APM score of the mother, prenatal exposure to paracetamol, antidepressants and benzodiazepines.

Table 3. Negative exposure: the association of exposure to NSAIDs before pregnancy and offspring attention problems reported by mothers

\begin{tabular}{llcl}
\hline Attention Problems & $\begin{array}{l}\text { N Exposed/ N Non- } \\
\text { exposed }\end{array}$ & $\begin{array}{l}\text { Unadjusted Mean Difference } \\
\text { in Attention Problems Score }\end{array}$ & $\begin{array}{c}\text { Adjusted Mean Difference in } \\
\text { Attention Problems Score } \dagger\end{array}$ \\
$\begin{array}{l}\text { Maternal report at } 1.5 \text { yrs. } \\
\text { Maternal report at } 3 \text { yrs. }\end{array}$ & $701 / 3689$ & $0.04(-0.11,0.18)$ & $-0.08(-0.22,0.06)$ \\
Maternal report at 5 yrs. & $979 / 3431$ & $0.08(-0.05,0.21)$ & $-0.06(-0.19,0.07)$ \\
& $0.05(-0.07,0.18)$ & $-0.08(-0.20,0.04)$ \\
\hline
\end{tabular}


Table 4. Negative outcome: The association of exposure to NSAIDs during pregnancy and offspring somatic problems reported by mothers

\begin{tabular}{llcr}
\hline Somatic complaints & $\begin{array}{l}\text { N Exposed/ N Non- } \\
\text { exposed }\end{array}$ & $\begin{array}{l}\text { Unadjusted Mean Difference } \\
\text { in Somatic Complaints Score }\end{array}$ & $\begin{array}{c}\text { Adjusted Mean Differe } \\
\text { Somatic Complaints Scon }\end{array}$ \\
Maternal report at 1.5 yrs. & $332 / 4024$ & $0.12(-0.05,0.29)$ & $0.09(-0.09,0.26)$ \\
Maternal report at 3 yrs. & $310 / 3804$ & $0.23(0.04,0.42)$ & $0.19(-0.01,0.38)$ \\
Maternal report at 5 yrs. & $370 / 4586$ & $0.24(0.05,0.44)$ & $0.13(-0.06,0.32)$
\end{tabular}

$\dagger$ Adjusted model, covariates: age and gender of the child, ethnicity, age, education and psychopathology of the mother, smoking and alcohol consumption during pregnancy, and APM score of the mother, prenatal exposure to paracetamol, antidepressants and benzodiazepines.

Table 5. Reporting bias: The association of exposure to NSAIDs during pregnancy and offspring attention problems reported by teachers and nonverbal IQ assessed by a research assistant

\begin{tabular}{llcc}
\hline Independent observations & $\begin{array}{l}\text { N Exposed/ N Non- } \\
\text { exposed }\end{array}$ & $\begin{array}{c}\text { Unadjusted Mean Difference } \\
\text { in Outcome Score }\end{array}$ & $\begin{array}{c}\text { Adjusted Mean Difference in } \\
\text { Outcome Score } \dagger\end{array}$ \\
$\begin{array}{l}\text { Teacher report of attention } \\
\text { problems at } 6 \text { yrs. }\end{array}$ & $276 / 3511$ & $-0.09(-1.16,0.98)$ & $-0.24(-1.23,0.76)$ \\
$\begin{array}{l}\text { Teacher report of somatic } \\
\text { problems at } 6 \text { yrs. }\end{array}$ & $274 / 3499$ & $-0.04(-0.16,0.09)$ & $-0.05(-0.17,0.08)$ \\
Non-verbal IQ assessment & $362 / 4729$ & $-0.07(-1.67,1.53)$ & $-0.32(-1.82,1.19)$ \\
\hline
\end{tabular}

†Adjusted model, covariates: age and gender of the child, ethnicity, age, education and psychopathology of the mother, smoking and alcohol consumption during pregnancy, and APM score of the mother, prenatal exposure to paracetamol, antidepressants and benzodiazepines. 
